\title{
EVOLUCIÓN DURANTE 44 AÑOS DE LAS CARACTERÍSTICAS MORFOLÓGICAS Y SOBREVIDA A LARGO PLAZO DE 304 PACIENTES CON CÁNCER GÁSTRICO INCIPIENTE*
}

\author{
Drs. Attila Csendes J. ${ }^{1}$, Juan C. Díaz J. ${ }^{1}$, Maher Musleh K. ${ }^{1}$, \\ Enrique Lanzarini S. ${ }^{1}$, Italo Braghetto M. ${ }^{1}$, Marcelo Zamorano D. ${ }^{1}$ \\ 1 Departamento de Cirugía, Hospital Clínico Universidad de Chile. Santiago. \\ Chile.
}

\begin{abstract}
Early gastric cancer. A 44 years' experience in 304 patients

Background: Early gastric cancer has a good long term prognosis. Aim: To report a 44 years' experience in the management of early gastric cancer. Material and Methods: Retrospective and prospective analysis of patients with early gastric cancer, managed in three periods: group I from 1970 to 1989, group II from 1990 to 2003 and group III from 2004 to 2013. Tumor location in the stomach, macroscopic type, pathological type, lymph node involvement and long term survival were analyzed. Results: Three hundred four patients with a mean age of 62 years (170 males) were analyzed. Tumors were intramucosal and submucosal in $136(45 \%)$ and $168(55 \%)$ patients, respectively. Among group III proximal tumors and diffuse pathological types were more common. Five and 15 years survival were 88 and $72 \%$, respectively. Conclusions: During the study period, tumor location and pathological types changed. Patients had high survival rates.
\end{abstract}

Key words: Early gastric cancer, location, survival.

\section{Resumen}

Introducción: El cáncer gástrico es una patología predominante en Chile. El carcinoma incipiente constituye una etapa inicial con muy buen pronóstico a largo plazo. El objetivo del presente estudio fue analizar las características morfológicas del carcinoma incipiente en 3 períodos de tiempo, con un período total de 44 años en un mismo centro, además de revisar la bibliografía nacional. Material y Método: Estudio mixto, tanto retrospectivo como prospectivo, desde 1970 hasta el año 2013, dividiendo a los pacientes en 3 grupos: grupo I desde 1970 hasta 1989, grupo II desde 1990 hasta 2003 y grupo III, desde 2004 hasta 2013. Se analizaron las siguientes variables: localización en el estómago, tipo macroscópico, tipo histológico, disección linfonodal y sobrevida a largo plazo. Para los cálculos estadísticos se empleó el Test de Fisher y $\chi^{2}$. La sobrevida se analizó según el Test de Kaplan-Meier. Resultados: Hubo un total de 304 pacientes, que corresponden al 11,3\% del total de pacientes resecados en el mismo período de tiempo. Hubo 136 pacientes con carcinoma mucoso

*Recibido el 11 de junio de 2014 y aceptado para publicación el 7 de julio de 2014.

Los autores no refieren conflictos de interés.

Correspondencia: Dr. Attila Csendes J. acsendes@hcuch.cl 
(44,7\%) y 168 con carcinoma submucoso (55,3\%). Se observó un aumento estadísticamente significativo de la localización proximal del tumor. Hubo un aumento significativo del tipo histológico difuso en el III período analizado. La sobrevida a 15 años plazo fue mayor al 70\%. Conclusiones: En 44 años se apreció un cambio en la localización del carcinoma incipiente y en el tipo histológico. La sobrevida alejada a 15 años es muy buena.

Palabras clave: Cáncer gástrico, incipiente, morfología, sobrevida.

\section{Introducción}

El cáncer gástrico es el tumor maligno de mayor mortalidad anual en Chile, con algo más de 3.000 pacientes que fallecen al año. Si bien desde la década de los 70 hasta los 90 las cifras de mortalidad han ido paulatinamente descendiendo, desde hace más de una década se mantiene en cifras de 19 fallecidos por 100.000 habitantes. El año 2000 la tasa de mortalidad fue de $19,6 \%$, el año 2005 de $19 \%$ y el año 2011 de 18,9\% con un total de 3.237 defunciones por cáncer gástrico ${ }^{1}$. La única manera de mejorar esta cifra es lograr pesquisar a los pacientes en etapas iniciales de la enfermedad, llamada carcinoma incipiente, como ocurre en Japón y Corea, donde el 50 a $60 \%$ de los pacientes presenta un cáncer incipiente ${ }^{2,3}$.

Desde el regreso del autor principal (A.C.) de Japón en 1969 , donde participó en el $1^{\text {er }}$ curso de cáncer gástrico, se fue difundiendo en todo Chile este nuevo concepto. Desde 1980, cuando publicamos la primera casuística con 28 pacientes ${ }^{4}$, existen varias publicaciones en nuestro país sobre este tema ${ }^{5-18}$

El objetivo del presente estudio fue: $1^{\circ}$ analizar las variaciones de las características morfológicas del carcinoma incipiente en 3 períodos de tiempo, que comprenden un total de 44 años, en un gran número de pacientes que hemos atendido. $2^{\circ}$ revisar toda la bibliografía nacional sobre este tema para reconocer la valiosa contribución de los diferentes grupos quirúrgicos.

\section{Material y Método}

\section{Pacientes estudiados}

La presente revisión es un estudio mixto, tanto retrospectivo como prospectivo, desde 1970 hasta diciembre de 2013, lo que equivale a un lapso de 44 años. Hasta 2003 es un análisis retrospectivo, pero en marzo de 2004 se creó la Unidad de Oncología con nuestro Departamento a cargo de una E.U. como se ha publicado previamente ${ }^{19}$. Desde esa fecha todos los pacientes que ingresan son registrados en una planilla Excel especialmente diseñada para este carcinoma, junto con una fotocopia del análisis anatomo-patológico. La profesional a cargo controla 1 vez al año a los pacientes para conocer si están vivos y si no, determinar la fecha precisa y el motivo del fallecimiento. Se excluyen de este estudio a los pacientes con cáncer "intermedio", es decir, el que infiltra la capa muscular o subserosa.

\section{Método}

Se dividió a los pacientes en 3 grupos de acuerdo a períodos de tiempo.

- Grupo I: desde enero de 1970 a diciembre de 1989.

- Grupo II: desde enero de 1990 hasta diciembre de 2003.

- Grupo III: desde enero de 2004 hasta diciembre de 2013.

Se analizan principalmente las siguientes características morfológicas:

a. Localización del carcinoma incipiente en los 3 períodos de tiempo.

b. Tipo macroscópico del tumor.

c. Tipo histológico y en evolución a lo largo del período de estudio.

d. Tiempo de disección linfonodal y porcentaje de linfonodos comprometidos.

e. Sobrevida a largo plazo.

Para el análisis histológico se empleó tanto la clasificación japonesa de carcinoma incipiente como la clasificación de Lauren $^{20,21}$.

\section{Cálculo estadístico}

Para el análisis de significación estadística se empleó el Test de $\chi^{2}$ y el Test de Fisher, tomando un $\mathrm{p}<0,05$ como significativo.

Para el cálculo de sobrevida actuarial se empleó el Test de Kaplan-Meier.

\section{Resultados}

En la Tabla 1 se señala el total de pacientes con cáncer gástrico resecados entre 1970 y 2013, que corresponde a 2.680 pacientes, con un promedio anual de 60 pacientes. De ellos hubo 304 casos $(11,3 \%)$ con un carcinoma incipiente cuya proporción fue aumentado progresivamente en los períodos sucesivos de tiempo. La diferencia entre el I y III período fue estadísticamente significativa. La Tabla 2 muestra las características generales del grupo analizado, con un predominio de 1,2 hombres por 1 mujer, 
con una edad promedio de 62 años. Hubo un total de 136 pacientes con carcinoma mucoso $(44,7 \%)$ y 168 pacientes con carcinoma submucoso $(55,3 \%)$.

La Tabla 3 describe las variaciones de la localización del carcinoma incipiente en los 3 períodos de tiempo: se aprecia un aumento progresivo de la localización proximal y distal y una disminución en el tercio medio del estómago. El aumento de los casos

Tabla 1. Proporción de carcinoma gástrico incipiente en 3 períodos entre 1970 y 2013

\begin{tabular}{|lccc|}
\hline & $\begin{array}{c}\text { Total } \\
\text { resecados }\end{array}$ & $\begin{array}{c}\text { Total } \\
\text { incipientes }\end{array}$ & $\%$ \\
\hline I.- 1970-1989 & 1.420 & 98 & $6,9(\mathrm{~A})$ \\
II.- 1990-2003 & 845 & 123 & $14,5(\mathrm{~B})$ \\
III.- 2004-2013 & 415 & 83 & $20,0(\mathrm{C})$ \\
Total & 2.680 & 304 & 11,3 \\
\hline
\end{tabular}

A vs $\mathrm{C}=\mathrm{p}<0,01$

Tabla 2. Características generales del cáncer gástrico incipiente. $\mathrm{N}=304$

\begin{tabular}{|lclc|}
\hline & $\begin{array}{c}\text { Mucoso } \\
\mathbf{n = 1 3 6} \\
\mathbf{( 4 4 , 7 \% )}\end{array}$ & $\begin{array}{c}\text { Submucoso } \\
\mathbf{n = 1 6 8} \\
\mathbf{( 5 5 , 3 \% )}\end{array}$ & $\mathbf{p}$ \\
\hline $\begin{array}{l}\text { 1. Género } \\
\text { Masculino }\end{array}$ & $75(55,1 \%)$ & $95(56,5 \%)$ & n.s. \\
Femenino & 61 & 73 & \\
2. Edad & & & \\
-49 & $47(15,5 \%)$ & & \\
$50-64$ & $118(38,8 \%)$ & & \\
$65-79$ & $115(37,8 \%)$ & & \\
$>/=80$ & $24(7,9 \%)$ & \\
\hline
\end{tabular}

n.s.: no significativo. con cáncer incipiente ubicados en el fondo es estadísticamente significativo $(\mathrm{p}<0,02)$ al comparar el grupo III con el grupo I y el grupo III con el grupo II $(p<0,02)$. No hubo diferencias entre el grupo I y II.

La Tabla 4 señala los tipos macroscópicos del carcinoma incipiente. Predominan las formas de tipo excavado o ulcerado sobre las lesiones de tipo solevantado, siendo esta diferencia estadísticamente significativa $(\mathrm{p}<0,04)$.

La Tabla 5 muestra los tipos histológicos del carcinoma incipiente y las variaciones a lo largo del tiempo: El tipo intestinal comprende en global al $75 \%$ de los pacientes, estando el adenocarcinoma tubular presente en el $70 \%$. El carcinoma papilar y el mucinoso tienen una baja proporción. En $2^{\circ}$ lugar está el adenocarcinoma en anillo de sello. Tomando la clasificación de Lauren en 3 períodos, se aprecia una disminución del tipo intestinal y un aumento del carcinoma difuso al doble entre el $1^{\text {er }}$ y $3^{\text {er }}$ período. Este aumento del carcinoma difuso entre el $1^{\text {er }}$ y $3^{\text {er }}$ período fue estadísticamente significativo $(\mathrm{p}<0,01)$, al igual que entre el $2^{\circ}$ y $3^{\text {er }}$ período $(\mathrm{p}<0,04)$.

Tabla 3. Localización del carcinoma gástrico incipiente.

$$
\mathbf{N}=\mathbf{3 0 4}
$$

\begin{tabular}{|llll|}
\hline Período & Sitio tumor & \% & \\
$1970-1989$ & Antro & 36 & \\
$n=98$ & Cuerpo & 49 & \\
& Fondo & 15 & (A) \\
$1990-2003$ & Antro & 33 & \\
$n=123$ & Cuerpo & 42 & \\
& Fondo & 25 & (B) \\
$2004-2013$ & Antro & 44 & \\
$n=83$ & Cuerpo & 37 & \\
& Fondo & 29 & (C) \\
\hline
\end{tabular}

$A$ vs $B=p>0,38$. A vs $C=p<0,02$. B vs $C=p<0,02$.

Tabla 4. Tipo macroscópico del carcinoma gástrico incipiente. $\mathbf{N}=304$

\begin{tabular}{|lcccrc|}
\hline Tipo macroscópico & $\begin{array}{c}\text { Mucoso } \\
\mathbf{n = 1 3 6}\end{array}$ & $\begin{array}{c}\text { Submucoso } \\
\mathbf{n}=\mathbf{1 6 8}\end{array}$ & $\begin{array}{c}\text { Total } \\
\mathbf{n}\end{array}$ & \% \\
I & 18 & 34 & 52 & $17,1^{*}$ & \\
IIa & 17 & 17 & 34 & $11,2 *$ & $* 31,2 \%(\mathrm{~A})$ \\
I+IIa & 3 & 6 & 9 & $2,9 *$ & \\
IIb & 10 & 11 & 21 & $6,9 *$ & \\
IIc & 43 & 37 & 80 & $96,3 *$ & $* 61,8 \%(\mathrm{~B})$ \\
III & 19 & 11 & 30 & $16,5^{*}$ & \\
IIc+III & 14 & 36 & 50 & $9,2 *$ & \\
IIc+IIa & 12 & 16 & 28 & & \\
\hline
\end{tabular}

$A$ vs $B=p<0,04$. 
Tabla 5. Características histológicas del carcinoma gástrico incipiente. $N=304$

\begin{tabular}{|lccr|}
\hline 1. Tipo histológico & $\begin{array}{c}\text { Mucoso } \\
\mathbf{n = 1 3 6}\end{array}$ & $\begin{array}{c}\text { Submucoso } \\
\mathbf{n}=\mathbf{1 6 8}\end{array}$ & \multicolumn{1}{c|}{ Total } \\
AdenoCa papilar & 4 & 10 & $14(4,6 \%)$ \\
AdenoCa tubular & 96 & 118 & $214(70,4 \%)$ \\
AdenoCa mucinoso & 3 & 7 & $10,3 \%)$ \\
AdenoCa en anillo de sello & 33 & 33 & $66(21,7 \%)$ \\
2. Tipo Lauren & Intestinal & Difuso & \\
Período & & & \\
a. $1970-1989$ & $80(81,6 \%)$ & $18(18,4 \%)(A)$ & \\
n $=98$ & & $28(22,8 \%)(B)$ & \\
b. $1990-2003$ & $95(77,2 \%)$ & & \\
n $=123$ & & $30(36,2 \%)(C)$ \\
c. $2004-2013$ & $53(63,8 \%)$ & \\
$n=83$ & & \\
\hline
\end{tabular}

$A$ vs $B=p>0,5$. A vs $C=p<0,01$. B vs $C=p<0,04$.

Tabla 6. Disección linfonodal en carcinoma gástrico incipiente. $\mathbf{N}=304$

\begin{tabular}{|lccc|}
\hline I. $\mathbf{n}$ linfonodos & Mucoso & Submucoso \\
Resecados & $\mathrm{n}=136$ & $\mathrm{n}=168$ & Total \\
$<20$ & 61 & 82 & $143(47 \%)$ \\
$21-40$ & 52 & 63 & $115(38 \%)$ \\
$<=41$ & 23 & 23 & $46(15 \%)$ \\
II. $\mathbf{n}$ pacientes con & & & \\
Linfonodos $(+)$ & $6(4,4 \%)(\mathrm{A})$ & $25(14,9 \%)(\mathrm{B})$ & $31(10,2 \%)$ \\
N1 $(+)$ & 6 & 24 & \\
$\mathrm{~N} 2(+)$ & & 1 & \\
\hline
\end{tabular}

A vs $B=p<0,003$.

La disección linfonodal se describe en la Tabla 5. En todos los pacientes se realizó una disección ganglionar $\mathrm{N} 1+\mathrm{N} 2$. El promedio de linfonodos resecados fue de 24,8 por paciente. El número de pacientes con linfonodos $(+)$ fue de $4,4 \%$ en carcinoma mucoso y de $14,9 \%$ en carcinoma submucoso diferencia que fue significativa $(\mathrm{p}<0,003)$. La mortalidad operatoria fue de 7,1\% (7/98) entre $1970 \mathrm{y}$ 1989 , de $3,2 \%(4 / 123)$ entre 1990 y 2003 y de $0 \%$ desde el 2004.

La sobrevida hasta 15 años se muestra en la Tabla 7 , con un $95 \%$ de seguimiento. La sobrevida promedio a 5 años fue de $88 \%$ y a 15 años de $72 \%$. A 60 meses el cáncer mucoso tiene una sobrevida de $92 \%$ y el submucoso de $85 \%$ ( $\mathrm{p}<0,04)$. A los 15 años la sobrevida es similar. Entre 210 pacientes seguidos hasta 15 años, hubo 35 fallecidos, de los cuales 24 fallecieron por otras causas (cardíacas, respiratorias, accidentes, etc.) y sólo 11 (5\%) por causas relacionadas con cáncer, con recurrencia ganglionar o hepática en los casos con ganglios $(+)$.
Tabla 7. Sobrevida de pacientes con carcinoma gástrico incipiente

\begin{tabular}{|lccl|}
\hline Sobrevida & Ca. Mucosso & Ca. Submucoso & \\
\hline 5 años & $92 \%$ & $85 \%$ & $\mathrm{p}<0,04$ \\
15 años & $75 \%$ & $72 \%$ & $\mathrm{p}>0,8$ \\
\hline
\end{tabular}

\section{Discusión}

El presente estudio es la serie chilena más grande de pacientes con cáncer gástrico incipiente atendidos en un mismo centro hospitalario, en un período muy largo de 44 años, que refleja la experiencia mayoritaria del autor principal. Los resultados sugieren que: 1) hay un aumento progresivo de la incidencia del cáncer temprano; 2) hay un cambio significativo en la localización de este tumor; 3 ) hay un significativo aumento del tipo difuso en el estudio histológico; 4) la sobrevida a 15 años es muy elevada. Cuando 
en 1970 se comenzó a estudiar la posibilidad de diagnosticar un cáncer gástrico incipiente, hubo reportes de casos aislados pero sin casuística. El primer estudio de un número de casos fue reportado por nuestro grupo en 1980 con 28 pacientes ${ }^{4}$. Desde esa fecha ha habido un importante aumento en la proporción de casos nuevos diagnosticados y así en la última década hemos llegado a un $20 \%$, contrastando con el 6\% de los años iníciales. En la Tabla 8 se muestra el resumen de los datos publicados por todos los grupos quirúrgicos chilenos en referencia a este tema ${ }^{4-18}$. Deseamos destacar e insistir una vez más en la necesidad de mencionar y referir los resultados nacionales, en vez de sólo referirse a publicaciones internacionales, como un reconocimiento al esfuerzo de los grupos quirúrgicos chilenos. En la Tabla 8 se aprecia que lo reportado va desde $2,8 \%$ hasta $15,8 \%$ de cánceres incipientes dentro del total de cánceres gástricos resecados. El aumento de cánceres tempranos se puede explicar por muchos factores: mejor nivel de educación ciudadana y por lo tanto consulta más precoz, posibilidad de acceder a diagnóstico endoscópico muy esparcido y difundido en todo Chile (comparando con la década del 70 y 80), establecimiento de protocolo del plan AUGE, atenciones de salud más accesibles, etc.

El carcinoma temprano ha mostrado 2 cambios muy significativos, que son exactamente iguales o paralelos a lo que ha ocurrido en pacientes con cáncer avanzado y publicado previamente ${ }^{22}$ : a) hay un cambio en la localización gástrica, con un significativo aumento en la última década del cáncer en tercio superior y b) paralelo a esto, hay un aumento significativo en la proporción de carcinoma de tipo difuso y una disminución del tipo intestinal. El carcinoma de tipo difuso, en especial el de "anillo de sello" desde el punto de vista patogénico no está relacionado con el Helicobacter pylori y crece en mucosa previamente sana. Esto significa que los tumores originados en el área oxíntica con gran frecuencia son de tipo difuso. Por el contrario, los cánceres de tipo intestinal tienen una secuencia patogénica conocida que va desde la metaplasia intestinal, displasia y el adenocarcinoma, relacionado con la infección del H. pylori. Estos cambios epidemiológicos han ocurrido igual en los cánceres avanzados.

La sobrevida de pacientes con cáncer gástrico es muy elevada y sobre $90 \%$ a los 5 años. Es muy conocido que 3 factores pueden incidir en una mayor o menor sobrevida: a) si es mucoso o submucoso $0^{4,16}$; b) si hay o no compromiso linfático metastásico ${ }^{16-18}$; c) el tipo histológico, que en estudios anteriores ${ }^{17} \mathrm{y}$ el nuestro no mostró realmente una diferencia significativa, contrastando con otro estudio ${ }^{18}$, que mostró diferencia significativa en la sobrevida. Hay sólo

Tabla 8. Resumen de autores chilenos que han reportado casuísticas de pacientes con cáncer gástrico incipiente

\begin{tabular}{|c|c|c|c|c|}
\hline Autor & $\mathbf{n}$ & Mucoso/submucoso & $\begin{array}{l}\text { Linfonodos }+ \text { mucoso- } \\
\text { submucoso }\end{array}$ & \% total Ca. Gástrico Op. \\
\hline Csendes (1980) & 28 & $11 / 17$ & $10 \% / 18 \%$ & -- \\
\hline Burmeister (1981) & 38 & $23 / 16$ & -- & -- \\
\hline Smok (1982) & 38 & $16 / 22$ & $2 \%$ & 6,6 \\
\hline Fernández (1982) & 30 & -- & $6,6 \%$ & 4,0 \\
\hline Guzmán (1982) & 15 & $10 / 5$ & $0 \%$ & 15,8 \\
\hline Hernández (1983) & 14 & $6 / 8$ & $0 \% / 0 \%$ & 7,0 \\
\hline Llanos (1985) & 156 & 12 & & 7,6 \\
\hline Burmeister (1987) & 68 & $37 / 31$ & $3 \% / 14,7 \%$ & -- \\
\hline Pisano (1990) & 19 & $9 / 10$ & $5 \%$ & -- \\
\hline Csendes (1991) & 233 & $77 / 156$ & $4,8 \% / 12 \%$ & 8,6 \\
\hline Lagos (1993) & -- & -- & -- & 2,8 \\
\hline Valenzuela (2004) & 14 & -- & -- & 13,7 \\
\hline Butte (2008) & 105 & $64 / 41$ & $7,8 \% / 22 \%$ & 10,0 \\
\hline Ceroni (2011) & 161 & $76 / 85$ & $2,9 \% / 11,5 \%$ & 10,0 \\
\hline Tapia (2011) & 106 & $47 / 59$ & $4 \% / 12 \%$ & 15,0 \\
\hline Csendes (2014) & 304 & $136 / 168$ & $4 \% / 15 \%$ & 11,3 \\
\hline
\end{tabular}


un estudio chileno con 10 años de seguimiento ${ }^{17}$, obteniendo resultados muy similares a los nuestros.

Nuestro protocolo actual incluye la realización de gastrectomía total en pacientes con carcinoma de tipo difuso y una gastrectomía subtotal sólo en pacientes con cáncer de tipo intestinal localizado en antro o cuerpo, en el que se asegura por lo menos un margen oral de $3 \mathrm{~cm}$ libre de tumor. Realizamos una disección linfonodal D2 de rutina. Por otra parte, se ha incorporado la cirugía laparoscópica en este tipo de pacientes, habiéndose realizado un número interesante de intervenciones que serán reportadas en otra publicación.

\section{Referencias}

1. DEIS (Departamento de Estadística e Información de Salud), Ministerio de Salud, 2011.

2. Lee JL, Kim W. Clinical experience of 528 laparoscopic gastrectomies on gastric cancer in a single institution. Surgery 2013;153:611-8.

3. Maehara Y, Kakegi J, Takahashi I, Azawaka C. Time trends of surgical treatment out the prognosis for Japanese patients with gastric cancer. Brit J Cancer 2000;83:986-91.

4. Csendes A, Smok G, Velasco N, Godoy M, Medina E, Braghetto I. Cáncer gástrico incipiente e intermedio. Análisis clínico y sobrevida de 51 casos. Rev Med Chile 1980;108:1011-5.

5. Burmeister R, Apablaza S, Covacevich S, Fernández M. Cirugía del cáncer gástrico incipiente. Rev Chil Cir. 1981;33:301-2.

6. Smok G, Csendes A. Carcinoma gástrico incipiente, intermedio y avanzado: características morfológicas. Rev Med Chile 1982;110:345-50.

7. Fernández E. Diagnóstico y tratamiento del cáncer gástrico incipiente e intermedio. Rev Chil Cir. 1982;34:192-7.

8. Guzmán S, Llanos O, Duarte I. Carcinoma gástrico incipiente. Rev Chil Cir. 1982;34:159-62.

9. Hernández F, Herve L, González R, Flisflisch H. Cáncer gástrico incipiente. Rev Chil Cir. 1983;35:181-3.

10. Llanos S, Valdés E, Tapia A. Cáncer gástrico en el Hospital Regional de Talca. Rev Med Chile 1985;113:213-7.
11. Burmeister R, Fernández H, Covacevich S. Experiencia de 15 años en el manejo del cáncer gástrico incipiente. Rev Chil Cir. 1987;39:23-6.

12. Pisano R, Venturelli A. Cáncer gástrico incipiente: experiencia del Hospital John Kennedy en Valdivia, período 1976-1989. Rev Med Chile. 1990;118:1111-5.

13. Csendes A, Smok G, Braghetto I, Medina E, Pizurno D, Guzmán S. Cáncer gástrico incipiente e intermedio. Aspectos anatomopatológicos y quirúrgicos. Estudio cooperativo entre 13 hospitales. Rev Med Chile 1991;119:1128-35.

14. Lagos D, Valenzuela L, Solano N, Góngora C, Pérez $\mathrm{F}$, Anabalon S. Interacción clínico-epidemológica del cáncer gástrico: provincias de Quillota y Petorca. Rev Chil Cir. 1993;45:546-50.

15. Valenzuela E, Martínez R, Sharp A, Naguira C, Roblero JP. Interposición del asa tipo Herley-Louguino en gastrectomía total: experiencia en el Hospital San Camilo de San Felipe. Rev Chil Cir. 2004;56:449-52.

16. Butte JM, Torres J, Viviani S, Duarte I, Crovari F, Guzmán S. Sobrevida alejada de pacientes operados por cáncer gástrico incipiente. Rev Med Chile 2008; 136;1424-30.

17. Ceroni M, García C, Benavides C, Covacevich S, Rubilar P, Cid H. Seguimiento mayor a 10 años de pacientes operados por cáncer gástrico incipiente. Rev Chil Cir. 2011;63:591-8.

18. Tapia O, García P, Manterola C, Villaseca H, Araya JC, Roa JC. Factores morfológicos asociados al pronóstico de pacientes operados por cáncer gástrico incipiente. Rev Chil Cir. 2011;63:162-9.

19. Cortés S, Csendes A, Yarmuch J. Resultados de la unidad de oncología del Departamento de Cirugía de un Hospital Universitario (2004-2010). Rev Chil Cir. 2011;63:534-37.

20. Japanese Gastric Cancer Association. Gastric cáncer guidelines. 3zd Edition, Ed. Kanchara Shumppoi, Tokyo 2010.

21. Lauren P. The two histological main types of gastric carcinoma: diffuse and so called intestinal type carcinoide. Acta Pathol Microbiol Scand. 1965;64:31-49.

22. Csendes A, Smok G, Medina E, Salgado I, Rivera R, Quitral M. Características evolutivas del cáncer gástrico 1958-1990. Rev Med Chile 1992;120:36-42. 